\title{
O PAPEL DAS INSTITUIÇÕES DE ENSINO SUPERIOR NA PREVENÇÃO DAS DOENÇAS IMUNOPREVENÍVEIS
}

\author{
THE ROLE OF HIGHER EDUCATIONAL INSTITUTIONS IN PREVENTING IMMUNEPREVENTABLE DISEASES
EL PAPEL DE LAZ INSTITUCIONES DE ENSEÑAZA UNIVERSITÁRIA EN LA PREVENIÓN DE LAS ENFERMIDADES INMUNOPREVENIBLES

\section{Silvana de Lima Vieira dos Santos ${ }^{1}$, Adenícia Custódia Silva e Souza ${ }^{2}$, Anaclara Ferreira Veiga Tipple ${ }^{2}$, Joaquim Tomé de Souza ${ }^{3}$}

\begin{abstract}
RESUMO: Estudo descritivo, realizado com alunos do último ano dos cursos de graduação da área de saúde, que objetivou caracterizar a atuação das Instituições de Ensino Superior (IES) na orientação do graduando quanto à vacinação, às doenças imunopreveníveis de importância para os Profissionais da Área de Saúde (PAS), em Goiás. Realizamos o teste piloto e com auxílio do software Epi-info, versão 2004, calculamos um $n=651$, o que corresponde a $54,0 \%$ da população de estudo, com intervalo de confiabilidade de 95\%. Foram observados os aspectos ético-legais. Os dados foram obtidos por meio de questionário, apresentados em figuras e tabelas e analisados por meio de estatística descritiva. Afirmaram ter sido orientados sobre as vacinas $92,0 \%$ dos estudantes. A informação foi obtida por meio de disciplina curricular em 32,3\%, e da direção da faculdade em $13,6 \%$ das citações. Reconhecem como vacinas recomendadas aos PAS: a anti- hepatite B em 94,8\%, a anti-difteria e tétano em $77,3 \%$ das citações. Embora as IES em Goiás estejam abordando esta temática, a fazem de forma fragmentada e pouco consistente.
\end{abstract}

PALAVRAS CHAVES: Exposição Ocupacional; Saúde do Trabalhador; Imunização.

ABSTRACT: This is a descriptive study peformed with the undergraduating students (last graded) in the health field. We aimed at characterizing the performance of the Higher Educational Institutions (HEI) in orientating such students concerning vaccination, immunepreventable diseases of high importance to heath care professionals (HCP), in Goiás. A sample test was carried out using Epi-Info 2004 Software, $n=651$, which corresponds to $54,0 \%$ of the studied population, reliability interval of $95 \%$. The ethical-legal aspects of the research were observed. Data was collected through questionnaire, shown in figures and tables, and assessed by descriptive statistics. Of the students, 92, 0\% reported having been orientated towards vaccination. Information was obtained by the means of curricular subject in $32,3 \%$, and the faculty directors accounting for $13,6 \%$ of reports. Vaccines which are known as recommended to such professionals are: anti-hepatitis B in 94,8\%, antidiphtheria and anti-tetanus in $77,3 \%$ of the citations. Although the HEI in Goiás have been discussing such issue, they do that in a fragmented and little consistent way.

KEY WORDS: Occupational Exposure; Occupational Health; Immunization.

RESUMEN: Estudio descriptivo realizado con alumnos del último año de estudios, de los cursos de graduación en el area de la salud, tuvo como objetivo la actuación de las Instituciones de Enseñanza Superior dando orientación al graduando en lo que se refiere a la vacunación de las enfermidades inmunoprevenibles importantes para los profesionales del area de la salud (PAS), en Goiás. Realizamos el test modelo y con el auxilio del software Epi-info, versão 2004, calculamos un $n=651$, lo que corresponde a $54,0 \%$ de la población estudiada, con un intervalo de confiabilidad de $95 \%$. Los aspectos éticos-legales fueron observados. Los datos fueron obtenidos mediante un cuestionario, presentados en figuras y tablas, analizados mediante estadística descriptiva. Confirmaron haber sido orientados sobre las vacunas $92,0 \%$ de los estudiantes. La información fue obtenida mediante disciplina del currículo de estudios en 32,3\% y en la dirección de la facultad en un $13,6 \%$ de las citaciones. Son reconecidas como vacunas recomendadas a los PAS: la anti-hepatitis B en $94,8 \%$, la anti-difteria y antitetánica en $77,3 \%$ de las citaciones. Aunque las IES en Goiás estén abordando este tema, lo realizan de forma fragmentada y de poca consistencia.

PALABRAS CLAVES: Exposición Ocupacional; Salud Laboral; Inmunización.

\footnotetext{
${ }^{1}$ Enfermeira. Especialista em Enfermagem em Infectologia. Mestre em Enfermagem. Professor da Universidade Salgado de Oliveira - Campus Goiânia, Goiás. e-mail: silvanalvs@hotmail.com

${ }^{2}$ Enfermeira. Doutora em Enfermagem. Professor Adjunto da Faculdade de Enfermagem da Universidade Federal de Goiás. Goiânia, Goiás. E-mail: adenicia@fen.ufg.br

3 Médico. Doutor em Fisiologia. Professor Titular da Faculdade de Enfermagem da Universidade Federal de Goiás. Goiânia, Goiás. E-mail tome@superig.com.br
} 


\section{INTRODUÇÃO}

Os Profissionais da Área de Saúde (PAS) estão expostos a vários tipos de riscos ocupacionais, sendo de maior impacto, o risco biológico.

O risco ocupacional biológico está presente ao manusear, de forma direta ou indireta, material orgânico de pacientes portadores de diversas patologias, sendo este, fonte de transmissão de microrganismos para pacientes e para outros profissionais (HOEFEL \& SCHNEIDER, 1997; QUEIROZ, 1998).

Os trabalhadores da área de saúde estão potencialmente expostos a diferentes tipos de agentes, tais como: vírus, bactérias, fungos, protozoários e ectoparasitas. O sangue e as vias aéreas representam as principais formas de contágio, seja por meio de acidentes com perfurocortantes, respingo de sangue em mucosas ou pela inalação de aerossóis ou gotículas (GOIÁS, 2003).

Ao relacionarmos este risco a estudantes, se torna mais preocupante, pois observamos que a prevenção e controle das doenças imunopreveníveis não têm sido tratadas com a ênfase esperada, embora recomendada pelo CDC (CDC, 2001) e Ministério da Saúde (BRASIL. MS, 2004).

TIPPLE (2000), ao investigar o estado vacinal de alunos de Odontologia de Goiânia, encontrou um alto percentual de adesão às vacinas que integram 0 esquema preconizado para a infância, chamando a atenção o fato de $6,4 \%$ destes alunos não referirem imunização anti-hepatite B, embora tenha sido implantada na Faculdade uma campanha de vacinação, após o ingresso desses alunos. Neste mesmo estudo, identificou-se ainda que a hepatite $B$ não é a doença infecto-contagiosa que mais os preocupa, sendo citada a hepatite B por $58,0 \%$ dos alunos, enquanto $91,1 \%$ referiram a AIDS.

CARVALHO et al (1998), em um estudo sobre hepatite $\mathrm{B}$ com o objetivo de identificar o perfil de proteção em estudantes dos cursos de Medicina, Odontologia e Enfermagem da Universidade Federal de Pernambuco (UFPE), verificaram que $49,0 \%$ dos entrevistados haviam recebido a vacina anti-hepatite $B$; $32,4 \%$ afirmaram não ter recebido e $18,5 \%$ não sabiam informar, ou seja, $51,0 \%$ dos estudantes estavam com baixo nível de proteção e com risco aumentado de adquirirem infecção. Os autores ressaltam a necessidade de se tornar obrigatória a comprovação da vacinação no ato da matrícula, pois somente por meio deste procedimento poderá ser garantido que os mesmos se protejam.

Em estudo realizado por REYN et al (2001), cujo objetivo foi analisar reações de teste de sensibilidade com proteínas derivadas do Mycobacterium tuberculosis e avium, entre PAS e estudantes de Medicina, identificaram que dos 395 estudantes somente $5,0 \%$ haviam recebido a BCG e do total geral de sujeitos (784), somente $3,0 \%$ haviam recebido a vacina BCG.

SOARES et al (2004), verificaram a prevalência da prova tuberculínica positiva, entre alunos da Faculdade de Medicina de Campos (RJ), e observaram que as taxas foram crescentes, de acordo com o progredir das atividades práticas com 13,1\% em alunos do último ano. Ressaltam que, as políticas públicas no Brasil priorizam a detecção do paciente bacilífero e do tratamento adequado, e pouca ou nenhuma ênfase é dada à biossegurança, no que se refere à $T B$, dos estudantes de Enfermagem e Medicina, e recomendam a realização de estudos pelo Ministério da Saúde, para validar a indicação de revacinação por BCG, bem como implantação de políticas nas Faculdades da área de saúde.

MALLET \& BUCCI (1994), realizaram um estudo para identificar a situação vacinal de estudantes de Farmácia, nos Estados Unidos, e verificaram que $53,0 \%$ das escolas ofereciam a vacina MMR (sarampo, caxumba e rubéola), 51,0\% a vacina contra tétano, $44,0 \%$ a vacina contra a hepatite $B$, e $5,0 \%$ outras vacinas. E concluíram que muitas Escolas de Farmácia não aderem às recomendações sobre imunização, fornecidas pelos CDC, para os PAS.

STACHDRA et al (2005), analisaram a situação vacinal dos graduandos do curso de Enfermagem e Fisioterapia das Faculdades Integradas Guarulhos e identificaram que $44,7 \%$ dos graduandos estavam com as vacinas atrasadas.

Independente da maneira como tem sido tratada esta temática nas instituições, espera-se que durante a formação acadêmica o aluno seja preparado quanto a medidas de promoção da saúde e prevenção de agravos, para si e para o cliente, podendo assim atuar de forma consciente e transformadora da realidade concreta.

A adesão a medidas ou programas de controle de infecção, só se concretiza quando há a compreensão de suas bases, sendo a educação um elemento fundamental neste processo (APECIH, 1998; BOLYARD et al, 1998).

Percebemos, por meio dos estudos com acadêmicos de cursos da área de saúde, que as instituições de ensino não têm abordado de forma efetiva, a prevenção e controle das doenças infecciosas. Entretanto, estes estudos trazem à luz a discussão da não-adesão à imunização e a falta de políticas nas IES, para que sejam alcançados maiores percentuais de graduandos vacinados. 
Visando mudanças no cenário nacional, e na tentativa de garantir maiores coberturas vacinais, no ano de 2004, o Ministério da Saúde do Brasil com a Portaria $n^{\circ} 597$, torna obrigatório em todo território nacional, para efeito de matrícula em Universidades, o comprovante de vacinação atualizado, de acordo com o calendário de vacinação e faixa etária estabelecidos pelo Programa Nacional de Imunização (BRASIL. MS, 2004)

\section{OBJETIVO}

Caracterizar a atuação da Instituição de Ensino Superior na orientação do graduando quanto à vacinação às doenças imunopreveníveis de importância para os PAS.

\section{METODOLOGIA}

Estudo descritivo sobre a atuação das Instituições de Ensino Superior no Estado de Goiás, na orientação dos graduandos de cursos da área de saúde, realizado, no período de abril a junho de 2005.

A população e amostra foram constituídas por alunos dos cursos da área de saúde, credenciados/autorizados pelo Ministério da Educação e Cultura (MEC), no Estado de Goiás, obtivemos 31 cursos em funcionamento, onde participaram do estudo 19 , os quais tinham alunos cursando o último ano/semestre em 2005.

Realizamos o teste piloto e com auxílio do software Epi-info, versão 2004, calculamos um $n=651$, o que corresponde a $54,0 \%$ da população, com intervalo de confiabilidade de $95 \%$. Foram observados os aspectos ético-legais da pesquisa. Os dados foram obtidos por meio de questionário, que foi validado por três juízes. A aplicação do instrumento foi em sala de aula. O banco de dados foi estruturado e processado no programa Epi-info, versão 2004 (CDC, 2004) e os dados apresentados em figuras e tabelas e analisados por meio de estatística descritiva.

\section{RESULTADOS E DISCUSSÃO}

Participaram do estudo 777 acadêmicos de cursos da área de saúde o que corresponde a $68,5 \%$ dos graduandos matriculados no último ano/período dos cursos em 2005 (Tabela 1), superando assim o quantitativo inicialmente esperado que era de 651 estudantes, ou seja, 54,0\%.

Tab.1 Distribuição de graduandos dos cursos da área de saúde que participaram do estudo, em relação ao número de alunos matriculados no último ano de cada curso em 2005. Goiás, 2005

\begin{tabular}{lccc}
\hline Cursos & $\begin{array}{c}\mathbf{N}^{\mathbf{0}} \text { de alunos } \\
\text { matriculados }\end{array}$ & $\begin{array}{c}\mathbf{N}^{\mathbf{0}} \text { de alunos } \\
\text { participantes }\end{array}$ & $\begin{array}{c}\text { Porcentagem de participantes } \\
\text { Odontologia }\end{array}$ \\
Biomedicina & 120 & 107 & $89,1 \%$ \\
Farmácia & 75 & 59 & $78,6 \%$ \\
Medicina & 231 & 772 & $74,4 \%$ \\
Fonoaudiologia & 110 & 76 & $69,0 \%$ \\
Terapia Ocupacional & 74 & 50 & $67,5 \%$ \\
Fisioterapia & 52 & 35 & $67,3 \%$ \\
Enfermagem & 160 & 96 & $60,0 \%$ \\
\hline TOTAL & 312 & 182 & $58,3 \%$ \\
\hline
\end{tabular}

Quanto à distribuição dos graduandos estudados de acordo com o curso, na Tabela 2, observa-se que a categoria com maior representatividade é a de Enfermagem com $182(23,4 \%)$ dos sujeitos, seguida pela Farmácia com $172(22,1 \%)$ e a Odontologia com
107 (13,8\%). Este fato se deve a um maior número de cursos de Enfermagem, em relação aos demais, pois no Estado de Goiás existem dez cursos de Enfermagem.

Tab. 2 Distribuição da freqüência e porcentagem de graduandos estudados por cursos da área de saúde. Goiás, 2005

\begin{tabular}{|l|c||c|}
\hline Cursos & Freqüência & Porcentagem \\
\hline Enfermagem & 182 & $\mathbf{2 3 , 4 \%}$ \\
\hline Farmácia & 172 & $\mathbf{2 2 , 1 \%}$ \\
\hline
\end{tabular}


SANTOS, Silvana de Lima Vieira dos; SOUZA, Adenícia Custódia Silva e; TIPPLE, Anaclara Ferreira Veiga; SOUZA, Joaquim Tomé de. O PAPEL DAS INSTITUIÇÕES DE ENSINO SUPERIOR NA PREVENÇÃO DAS DOENÇAS IMUNOPREVENÍVEIS. Revista Eletrônica de Enfermagem, $v$. 08, n. 01, p. 91-98, 2006. Disponível em http://www.fen.ufg.br/revista/revista8 1/original 12.htm

\begin{tabular}{|l||c||c|}
\hline Odontologia & 107 & $\mathbf{1 3 , 8 \%}$ \\
\hline Fisioterapia & 96 & $12,4 \%$ \\
\hline Medicina & 76 & $9,8 \%$ \\
\hline \hline Biomedicina & 59 & $7,6 \%$ \\
\hline Fonoaudiologia & 50 & $6,4 \%$ \\
\hline Terapia Ocupacional & 35 & $4,5 \%$ \\
\hline Total & $\mathbf{7 7 7}$ & $\mathbf{1 0 0 , 0 \%}$ \\
\hline
\end{tabular}

A idade dos acadêmicos variou de 20 a 55 anos de idade, com média de 34,7 anos. A faixa etária com maior predominância é de 20 a 30 anos com 650 $(86,1 \%)$ dos sujeitos, o que demonstra uma população jovem, em sua maioria, freqüentando os cursos superiores na área de saúde.

O papel das IES na prevenção de doenças imunopreveníveis
As Instituições de Ensino Superior tem papel primordial na prevenção e controle das doenças imunopreveníveis, pois é durante a formação acadêmica que se fundamentam conceitos e é construído o conhecimento.

Ao verificarmos sobre a orientação acerca da imunização durante a graduação, apresentada na Figura 1, $710(92,0 \%)$ dos estudantes afirmou ter sido orientada, o que caracteriza a participação das IES na formação do graduando quanto à prevenção de doenças infecciosas.

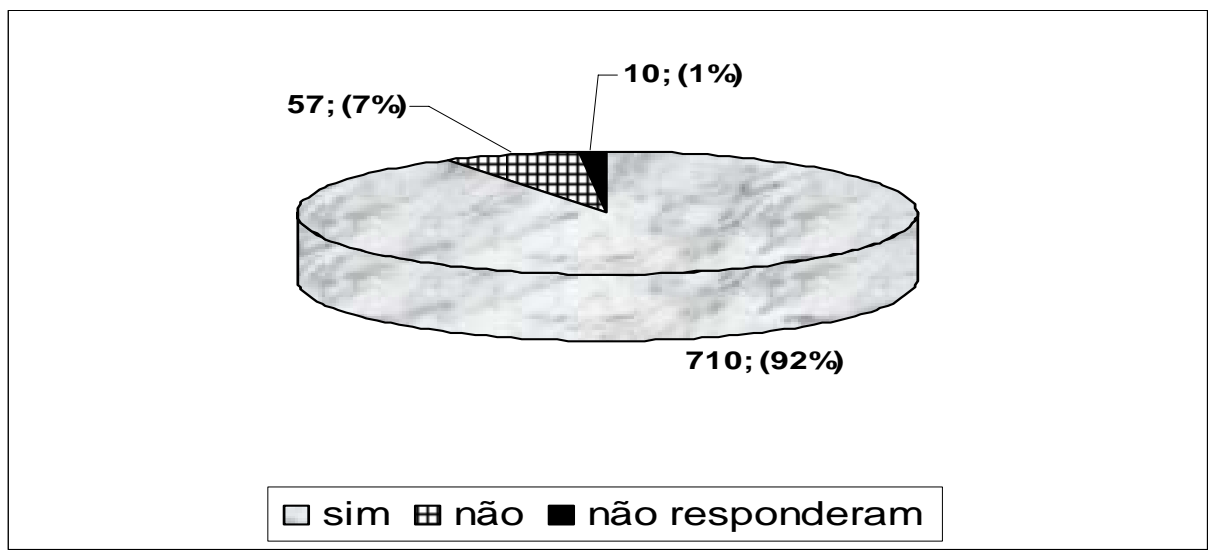

Fig. 1 Distribuição da porcentagem de graduandos dos cursos da área de saúde, segundo o recebimento de orientação acerca da necessidade de imunização. Goiás, 2005

Apesar de ser significativo o percentual de alunos orientados sobre imunização como prevenção de doenças, observamos na Tabela 3, que em apenas dois cursos, Medicina e Biomedicina todos os graduandos relataram ter recebido esta orientação.

O conhecimento construído na academia é fundamental e tende a ser aplicado e repetido pelo trabalhador, quando inserido no mercado de trabalho
(AZAMBUJA et al, 2004). Portanto, durante a graduação, os egressos devem ter "construído a compreensão dos aspectos teóricos e práticos da prevenção e controle das infecções relacionadas aos serviços de assistência a saúde, pois constituirão elementos de uma atuação profissional coerente" (MELO, 2005 p.69).

Tab.3 Distribuição dos graduandos da área de saúde, orientados a se imunizarem durante a graduação, segundo o curso. Goiás, 2005

\begin{tabular}{|l|c|c|c|}
\hline CURSO & Total de alunos & Orientados & \% \\
\hline Biomedicina & 59 & 59 & 100,0 \\
\hline Medicina & 76 & 76 & 100,0 \\
\hline Fisioterapia & 96 & 95 & 98,9 \\
\hline
\end{tabular}


SANTOS, Silvana de Lima Vieira dos; SOUZA, Adenícia Custódia Silva e; TIPPLE, Anaclara Ferreira Veiga; SOUZA, Joaquim Tomé de. O PAPEL DAS INSTITUIÇÕES DE ENSINO SUPERIOR NA PREVENÇÃO DAS DOENÇAS IMUNOPREVENÍVEIS. Revista Eletrônica de Enfermagem, $v$. 08, n. 01, p. 91-98, 2006. Disponível em http://www.fen.ufg.br/revista/revista8 1/original 12.htm

\begin{tabular}{|l|c|c|c|}
\hline Odontologia & 107 & 10 & 97,1 \\
\hline Terapia Ocupacional & 35 & 34 & 97,1 \\
\hline Enfermagem & 182 & 164 & 90,1 \\
\hline Fonoaudiologia & 50 & 43 & 86,0 \\
\hline Farmácia & 172 & 135 & 78,4 \\
\hline
\end{tabular}

As vacinas recomendadas durante a graduação, citadas com maior freqüência (Tabela 4), foram a vacina anti-hepatite B por 478 (61,5\%), anti-difteria e tétano por 457 (58,8\%), anti-amarílica por $223(28,7 \%)$ e contra hepatite por $134(17,2 \%)$ dos graduandos.

Tab. 4 Distribuição da freqüência e porcentagem de vacinas recomendadas aos graduandos dos cursos da área de saúde, durante a graduação. Goiás, 2005

\begin{tabular}{|l|c|c|}
\hline Vacinas & Fr & \% \\
\hline anti-hepatite B & 478 & $\mathbf{6 1 , 5}$ \\
\hline anti-difteria e tétano & 457 & $\mathbf{5 8 , 8}$ \\
\hline anti-amarílica & 223 & $\mathbf{2 8 , 7}$ \\
\hline anti-hepatite & 134 & $\mathbf{1 7 , 2}$ \\
\hline anti-rúbeola & 119 & 15,3 \\
\hline BCG & 83 & 10,6 \\
\hline Outras & 23 & 2,9 \\
\hline anti-influenza & 15 & 1,9 \\
\hline não se lembra & 12 & 1,5 \\
\hline
\end{tabular}

Destacamos nesta tabela que 23 graduandos $(2,9 \%)$ citaram outras vacinas, dentre as quais a anticaxumba e a anti-sarampo, mostrando não serem relevantes dentro da estatística descrita. Estes dados demonstram que, apesar das instituições de ensino orientarem seus alunos sobre a imunização, este ensino tem sido frágil, não explicitando quais vacinas devem ser recomendadas para os profissionais da área de saúde, mesmo estando preconizado pela Comissão Estadual de Controle de Infecção Hospitalar (CECIH), (GOIÁS, 2003).

Identificamos que, apenas uma unidade de ensino de nossa amostra, certifica no ato da matrícula se o aluno está com o cartão de vacinas em dia e, caso não esteja, solicita começar o esquema vacinal e apresentar o comprovante. No ano de 2004, com a Portaria 597, o Ministério da Saúde torna obrigatória a vacinação como requisito para matrícula em instituições de ensino (BRASIL. MS, 2004). O descumprimento desta portaria pode expor os graduandos, que em alguns cursos começam precocemente a prática clínica.

Estudo realizado por COOK et al (1993), em colégios e universidades americanas, identificou que $55,0 \%$ destas instituições de ensino solicitam o comprovante de vacinação, no ato da matrícula.

Dentre as 19 instituições estudadas, observamos que somente três IES disponibilizam as vacinas na própria instituição e as demais orientam seus graduandos a procurarem as unidades básicas de saúde dos respectivos Municípios. Verificamos que dezoito unidades recomendam aos graduandos, que se vacinem antes de iniciarem as atividades práticas, sem, entretanto, comprovação posterior. Há mais de dez anos, já havia esta preocupação (COOK et al, 1993) e como pode ser verificado, não houve mudanças significantes no decorrer destes anos, mesmo se tratando de diferentes realidades.

Apresentamos, na Tabela 5, as fontes de informação dos graduandos sobre as vacinas recomendadas aos PAS. As mais citadas foram: disciplina curricular com $293(37,7 \%)$ seguida pela direção da faculdade com 259 (33,3\%), sendo a instituição de ensino, a fonte dessas orientações em grande parte dos cursos.

O profissional da área de saúde, que se encontra em situação permanente de exposição ao sangue, além de se vacinar contra a hepatite $B$, recomenda-se a realização do teste anti-HBs, para conhecimento da resposta vacinal, tendo como objetivo conferir se o indivíduo adquiriu imunidade, e auxiliar na profilaxia pós-exposição ocupacional (APEClH, 1998; CONCEIÇẨO et al, 2000; CDC, 2001).

Identificamos, na Tabela 5, que a orientação para realizar o exame anti-HBs, ocorreu por meio de disciplina curricular, como citado por 251 alunos $(32,3 \%) ; 106(13,6 \%)$ citaram a direção da faculdade e $89(11,5 \%)$, por meio de colegas. Consideramos baixo o número de alunos orientados quanto à realização de 


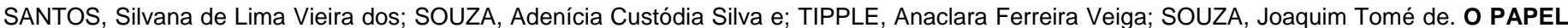
DAS INSTITUIÇÕES DE ENSINO SUPERIOR NA PREVENÇÃO DAS DOENÇAS IMUNOPREVENÍVEIS. Revista Eletrônica de Enfermagem, $v$. 08, n. 01, p. 91-98, 2006. Disponível em http://www.fen.ufg.br/revista/revista8 1/original 12.htm

um exame de tamanha importância, corroborando, mais uma vez, com as nossas indagações quanto à consistência da construção desse conhecimento pelos acadêmicos, no decorrer da graduação.

Ainda, em relação à disciplina curricular enquanto fonte de informação sobre as vacinas, identificamos que os graduandos dos cursos de Enfermagem e Farmácia foram os que afirmaram esta fonte, com maior freqüência, sendo 91 (36,2\%) e 57

Tab. 5 Distribuição da freqüência e porcentagem da fonte de orientação para as vacinas recomendadas aos alunos, durante a graduação, e para a realização de exame anti-HBs pós-vacina anti-hepatite B. Goiás, 2005

\begin{tabular}{|l|c|c|c|c|}
\hline \multirow{2}{*}{ Como tomou conhecimento } & \multicolumn{2}{|c|}{ Vacinas } & \multicolumn{3}{|c|}{ Anti-HBs } \\
\cline { 2 - 6 } & Freqüência & \% & Freqüência & \multicolumn{2}{c|}{ \% } \\
\hline Disciplina curricular & 293 & $\mathbf{3 7 , 7}$ & 251 & $\mathbf{3 2 , 3}$ \\
\hline Direção da Faculdade & 259 & $\mathbf{3 3 , 3}$ & $\mathbf{1 3 , 6}$ & $\mathbf{1 1 , 5}$ \\
\hline Com colegas de turma & 76 & 9,7 & 89 & 8,4 \\
\hline Eventos extra-curriculares & 61 & 7,8 & 66 & \\
\hline Campanha vacinal durante a graduação & 151 & 19,4 & 60 & \\
\hline Outros & 36 & 4,6 & 49 & \\
\hline Não recebeu esta informação & - & - & 41 & \\
\hline
\end{tabular}

Apesar da disciplina curricular se constituir, na maior fonte de informação, observamos uma diluição deste conteúdo em uma série de disciplinas, desde as do ciclo básico, comum a todas os cursos, como Bioquímica, Imunologia e Microbiologia, até disciplinas específicas de cada curso, não havendo consenso quanto a uma disciplina específica, sendo citadas aproximadamente 32 disciplinas diferentes. Em princípio, parece ser interessante este enfoque gradual e poderia denotar uma construção sólida deste conhecimento, não fosse a fragmentação existente, ainda hoje, no ensino, no qual cada disciplina funciona de forma estanque, sem integração interdisciplinar.

Espera-se que 0 ensino formal seja 0 responsável pela construção deste conhecimento, e verificamos que não corresponde nem a 50,0\% das citações, apesar de 92,0\% dos graduandos relatarem terem sido orientados sobre imunização.

MELO et al (2004), em um estudo realizado com estudantes do último ano de Enfermagem de quatro cursos, no Estado de Goiás, verificaram que a abordagem das medidas de prevenção e controle de infecção ocorre em associação às temáticas referentes à formação técnica, não existindo um consenso quanto a uma disciplina específica.

Em estudo realizado por TIPPLE (2000), com estudantes de Odontologia, no qual analisou a relação do processo de ensino com o controle de infecção, identificou que existe fragilidade sobre o conhecimento das medidas básicas de controle de infecção, relacionadas às atividades clínicas dos alunos, pois verificou uma multiplicidade de condutas utilizadas pelos alunos, resultantes da falta de padronização nas diferentes disciplinas e lacunas teóricas e práticas, reveladas nas ementas e conteúdos programáticos das disciplinas.
$(22,7 \%)$, respectivamente. Ao fazermos a relação de citações de acordo com a proporção de alunos, por curso, verificamos que ambos apresentam esta fonte em menos de 50\%, bem como os demais cursos. Reforçando que, embora a disciplina curricular esteja presente, não é a fonte principal de informação como acreditávamos.
TIPPLE et al (2003), afirmam que uma única disciplina não dará suporte suficiente para se trabalhar medidas preventivas, para o controle de infecção e que todos os docentes deverão estar envolvidos com os conteúdos sobre esta temática, pois se atribui ao corpo docente, das instituições formadoras, um peso importante na formação profissional.

$\mathrm{Na}$ verdade, os graduandos receberam algum conteúdo sobre imunização durante a graduação, porém ocorreu de forma compartimentada, e o aluno por si não foi capaz de agregá-lo a ponto de transformá-lo em uma prática no seu cotidiano, demonstrando que não existe interdisciplinaridade. Para VILELA \& MENDES (2003), a interdisciplinaridade é a interação entre duas ou mais disciplinas, em contexto de estudo e âmbito mais coletivo, que resulta em enriquecimento recíproco e transformação de suas metodologias.

Percebe-se ainda que, a maioria dos graduandos recebeu esta orientação por meio da direção da faculdade, eventos extra-curriculares, campanhas vacinais ou mesmo com colegas de turma (Tabela 5), o que nos leva a questionar a consistência das informações recebidas.

A imunização é parte essencial dos programas de controle de infecção em saúde ocupacional, pois asseguram ao trabalhador da área de saúde a redução nos riscos de aquisição a doenças imunopreveníveis, bem como redução no número de indivíduos e profissionais suscetíveis, reduzindo assim o risco de transmissão de doenças dos profissionais aos pacientes e vice-versa (PRABHAKAR et al, 2000).

Apesar das diferentes formas de orientação sobre imunização, quando solicitamos que os alunos relacionassem as vacinas recomendadas, para os PAS (Tabela 6), verificamos que 737 alunos $(94,8 \%)$ 
SANTOS, Silvana de Lima Vieira dos; SOUZA, Adenícia Custódia Silva e; TIPPLE, Anaclara Ferreira Veiga; SOUZA, Joaquim Tomé de. O PAPEL DAS INSTITUIÇÕES DE ENSINO SUPERIOR NA PREVENÇÃO DAS DOENÇAS IMUNOPREVENÍVEIS. Revista Eletrônica de Enfermagem, $v$. 08, n. 01, p. 91-98, 2006. Disponível em http://www.fen.ufg.br/revista/revista8 1/original 12.htm

reconhecem as vacinas anti-hepatite $\mathrm{B}, 601(77,3 \%)$ a

anti-difteria e tétano e $376(48,3 \%)$ a anti-amarílica.

Tab. 6 Distribuição dos graduandos da área de saúde em relação ao conhecimento das vacinas recomendadas, para os profissionais da área de saúde. Goiás, 2005

\begin{tabular}{|l|c|c|}
\hline Vacinas recomendadas aos PAS & Freqüência & \% \\
\hline anti-hepatite B & 737 & $\mathbf{9 4 , 8}$ \\
\hline anti-difteria e tétano & 601 & $\mathbf{7 7 , 3}$ \\
\hline anti-amarílica & 376 & $\mathbf{4 8 , 3}$ \\
\hline BCG & 261 & $\mathbf{3 3 , 5}$ \\
\hline anti-rubéola & 258 & $\mathbf{3 3 , 2}$ \\
\hline anti-influenza & 149 & $\mathbf{1 9 , 1}$ \\
\hline anti-sarampo & 92 & 11,8 \\
\hline Outras & 8 & 1,0 \\
\hline
\end{tabular}

Identificamos ainda que, para as demais vacinas, menos de $35,0 \%$ dos estudantes sabem quais são as recomendadas. Isto é preocupante, pois se trata de futuros profissionais a um passo de ingressarem no mercado de trabalho. Estes dados nos levam a refletir sobre a qualidade do ensino, sobre a temática de imunização dos PAS, enquanto medida eficaz no controle de doenças imunopreveníveis.

PEREIRA et al (2005) referem que, viabilizar o contato do estudante com todas as normas e legislações orientadoras e reguladoras de prevenção e controle de infecção é um importante caminho, e quanto mais precoce se fizer na graduação, maior a chance do futuro profissional se conscientizar e assimilar sobre a temática, devendo este conteúdo compor as políticas da instituição de formação profissional. Concordamos com os autores, pois somente por meio de mudanças nas políticas das IES alcançaremos o perfil desejado do egresso, quanto ao ensino da prevenção e controle de infecções.

\section{CONCLUSÃO}

As Instituições de Ensino Superior, no Estado de Goiás, no que se refere à imunização dos graduandos, precisam dispensar maior atenção a este tema, pois verificamos neste estudo que, embora estas trabalhem a temática do controle de infecção, a abordagem tem sido fragmentada e insuficiente, para que o aluno construa um corpo de conhecimento consistente e aplicável a realidade.

Ressaltamos que, o ensino da prevenção e controle de infecção deve ser adotado com seriedade pela IES e principalmente, pelos responsáveis pelas disciplinas que ministram as atividades práticas, peculiares às profissões da área de saúde. As IES precisam observar o exposto na portaria $n^{\circ} 597$ que trata da obrigatoriedade da imunização, para efeito de matrícula em universidades, em todo o território nacional (BRASIL. MS, 2004).
O ensino desta temática é antes de tudo, um compromisso ético, tanto das IES, quanto dos educadores. E consideramos que deva ser estabelecida, com urgência, uma política em que seja dada a ênfase necessária à prevenção de doenças infecciosas que se adquire ocupacionalmente, e de tanta importância para os profissionais da área de saúde.

\section{REFERÊNCIAS BIBLIOGRÁFICAS}

APECIH - ASSOCIAÇÃO PAULISTA DE ESTUDOS E
CONTROLE DE INFECÇÃO HOSPITALAR. Orientações para controle de infecção em pessoal da área da saúde. São Paulo, 1998. 94 p.

AZAMBUJA, E.P; PIRES, D.P; VAZ, M.R.C. Prevenção e controle de infecção hospitalar: as interfaces com o processo de formação do trabalhador. Texto Contexto Enfermagem, Florianópolis, v.13, n. especial, p. 79-86, 2004.

BOLYARD, E.A. et al. Guideline for infection control in healthcare personnel. Infection Control and Hospital Epidemioly, New Jersey, v.19, n. 6, p. 410-463, 1998.

BRASIL, Ministério da Saúde. Portaria $n^{\circ} 597$, de 8 de abril de 2004. Institui em todo território nacional os calendários de imunização de crianças, adolescentes, adultos e idosos, visando o controle, eliminação e erradicação das doenças imunopreveníveis por intermédio do Programa Nacional de Imunizações, vinculado ao Departamento de Vigilância Epidemiológica - DEVEP, da Secretaria de Vigilância em Saúde. Subsecretária de Informações. Brasília. 2004.[on line] Disponível em: http://dtr2001.saude.gov.br/sas/PORTARIAS/Port2004/ GM/GM-597.htm [Acesso em nov. de 2004].

CARVALHO, T.F.A et al. Hepatite B: perfil de proteção em estudantes dos cursos de Medicina, Odontologia e Enfermagem da UFPE. Revista do Instituto Materno Infantil de Pernambuco, Recife, v. 12, n .2, p .30-33, 1998. 
CENTER FOR DISEASE CONTROL AND PREVENTION - CDC. Guidelines for the management of occupational exposures to HBV, HCV and HIV and recomendations for postexposure prophylaxis. MMWR. Atlanta. v. 50. RR-11. 2001.

.Epi-info program version 3.3 of October 2004. Atlanta. 2004. [online] Disponível em: http://www.cdc.gov. [Acesso em nov. de 2004].

CONCEIÇÃO, F.M; CAVALCANTE, N.J.F; AYUB, M.A. Imunização em profissionais de saúde. In: FERNANDES, A.T; FERNANDES, M.O; FILHO, N.R. Infecção hospitalar e suas interfaces na área de saúde. v. 2. ATHENEU. São Paulo, 2000. p.1020-1025.

COOK, L.G et al. Prematriculation immunization requeriments of American colleges and universities. American Journal College Health Association, Evanston, v. 42, n. 3, p. 91-98, 1993.

GOIÁS, Secretaria de Estado da Saúde. Coordenação Estadual de Controle de Infecção Hospitalar. Programa de prevenção e assistência ao acidente profissional com material biológico. Goiânia, 2003. 24 p.

HOEFEL, H.H.K; SCHNEIDER, L. O profissional de saúde na cadeia epidemiológica. In: RODRIGUES, E.A.C; MENDONÇA, J.S; AMARANTES, J.M.B et al. Infecções Hospitalares: prevenção e controle. São Paulo: SARVIER,1997, p. 352-366.

MALLET, L.; BUCCI, K.K. Immunization requeriments for Pharmacy students. Annals of Pharmacotherapy, Cincinnati, v. 28, n.10, p. 1153-1158, 1994.

MELO, D.S. et al. Precauções padrão: conhecimento e crenças dos graduandos em enfermagem. In: IX Congresso Brasileiro de Controle de Infecção e Epidemiologia Hospitalar; Salvador, 2004. Anais. Salvador, 2004. CD-ROM.

MELO, D.S. Adesão dos enfermeiros às precauções padrão à luz do modelo de crenças em saúde. 2005. 191 p. Dissertação (Mestrado) - Faculdade de Enfermagem. Universidade Federal de Goiás, Goiânia. PEREIRA, M.S et al. A infecção hospitalar e suas implicações para o cuidar da enfermagem. Texto e Contexto Enfermagem, Florianópolis, v.14, n. 2, p. 250257, 2005.

PRABHAKAR, P. et al. Immunization of health care workers in the CARICOM countries. West Indian Medical Journal, Kingston, v. 49, n .4, p.353-355. 2000. QUEIROZ, M.C.B. Biossegurança. In: OLIVEIRA, A.C.; ALBUQUERQUE, C.P.; ROCHA, L.C.M. Infecções Hospitalares-abordagem, prevenção e controle. Rio de Janeiro: MEDSI, 1998, p.183-195.

REYN, C.F.V et al. Skin test reactions to mycobacterium tuberculosis purified protein derivate and mycobacterium avium sensitin among health care workers and medical students in the United States. International Journal Tuberculosis and Lung Disease, Paris, v .5, n.12, p.1122-1128, 2001.
SOARES, L.C.P; MELLO, F.C.Q; KRITSKI, A.L. Prevalência da prova tuberculínica positiva entre alunos da Faculdade de Medicina de Campos (RJ). Jornal Brasileiro de Pneumologia, Brasília, v. 30, n .4, p .350357. 2004.

STACHDRA, E.W et al. Análise da situação vacinal dos graduandos do curso de Enfermagem e de Fisioterapia das Faculdades Integradas Guarulhos. Revista da Sociedade Brasileira de Medicina Tropical, Brasília, v. 38, n.1, p. 256, 2005.

TIPPLE, A.F.V. As interfaces do controle de infecção em uma instituição de ensino odontológico. 2000. 177 p. Tese (Doutorado) - Escola de Enfermagem, Universidade de São Paulo, Ribeirão Preto.

TIPPLE, A.F.V. et al. O ensino do controle de infecção: um ensaio teórico-prático. Revista Latino-americana de Enfermagem, Ribeirão Preto, v. 11, n. 2, p. 245-250, 2003.

VILELA, E. M; MENDES, I.J. Interdisciplinaridade e saúde: estudo bibliográfico. Revista Latino-americana de Enfermagem, Ribeirão Preto, v. 11, n. 4, p. 525-531, 2003.

Texto original recebido em 21/02/2006

Publicação aprovada em 30/04/2006 